\title{
Zwischen Union und Devianz. Der Transfer religiöser Ideen im Raum der Personalunion
}

\author{
Manfred Jakubowski-Tiessen
}

I.

Am Anfang ging es um die Religion; genauer: um die Konfession. Es ging um die Frage, wie eine protestantische Erbfolge auf dem englischen Thron sicher gestellt werden könnte. Als Queen Anne nach achtzehn Fehl- oder Totgeburten im Jahre 1700 auch ihren einzigen noch lebenden Sohn im Alter von 11 Jahren infolge einer Pockenerkrankung verlor, war abzusehen, dass mit ihrem Tod die protestantische Linie des Hauses Stuart erlöschen würde. Aus diesem Grunde legte das englische Parlament $1701 \mathrm{im}$ Act of Settlement fest, dass nach dem Tod der Königin Anne das Recht der Thronfolge auf Sophie von der Pfalz, der protestantischen Kurfürstin von Hannover, übergehen sollte. Sophie stammte mütterlicherseits von den Stuarts ab; ihre Mutter Elisabeth war die Tochter Jakobs I. von England. Kurfürstin Sophie starb jedoch wenige Wochen vor Eintritt des Erbfalls, so dass 1714 ihr Sohn, Kurfürst Georg Ludwig, als Georg I. den britischen Thron bestieg und damit die 123-jährige Personalunion zwischen Großbritannien und Kurhannover begründete. Wie wichtig die konfessionelle Komponente bei dieser Erbfolge war, zeigt sich nicht zuletzt darin, dass die Thronfolge im Jahr 1714 von den englischen Zeitgenossen nicht als Hanoverian succession, sondern vornehmlich als Protestant Succession angesehen wurde. ${ }^{1}$

Mit der Thronfolge von 1714 kam England nun in den Besitz der Welfen, einer Dynastie, welche sich als Verfechterin protestantischer Interessen in den Jahrzehnten vor dem Act of Settlement nicht gerade hervorgetan hatte. Herzog Johann Friedrich, der Onkel des späteren Königs Georg I., war im Alter von 26 Jahren in Assisi zum Katholizismus konvertiert; allerdings verzichtete er nach Übernahme der hannoverschen Regierung im Jahr 1665 auf das ius reformandi, die Rekatholisierung seines Landes, weil er den Widerstand der Landstände fürchtete. Diese politische Rücksichtnahme

1 Vgl. Andrew C. Thompson, Britain, Hanover and the Protestant Interest, 1688-1756, Studies in early modern cultural, political and social history 3, Woodbridge 2006, S. 60, 167. 
hinderte ihn jedoch nicht daran - wenn auch sehr vorsichtig und nicht öffentlich die Pläne des römisch-katholischen Bischofs und kaiserlichen Gesandten Cristobal Royas y Spinola zu unterstützen, der sich um eine Reunion der Konfessionen, d.h. die Rückführung der Protestanten in die katholische Kirche bemühte und entsprechende Gespräche in Hannover führte. ${ }^{2}$ Mit dem Tod Johann Friedrichs schien aus kaiserlicher und römischer Sicht die Hoffnung auf eine weitere Ausbreitung des Katholizismus in Norddeutschland zunächst zu schwinden.

In gleicher Weise hatte Johann Friedrichs Bruder Ernst August, seit 1662 lutherischer Fürstbischof in Osnabrück und ab 1679 als Nachfolger seines Bruders Herzog von Braunschweig-Calenberg, bereits während seiner Osnabrücker Zeit über einen Konfessionswechsel mit dem kaiserlichen Hof verhandelt. Anders als bei seinem Bruder hatte der avisierte Übertritt zur katholischen Kirche bei Ernst August ausschließlich politische Motive. Er wäre zu einer Konversion bereit gewesen, wenn dadurch das Alternat auf dem Osnabrücker Bischofssitz, also die wechselnde Herrschaft zwischen katholischem und protestantischem Fürstbischof, aufgehoben und das Hochstift Osnabrück rechtsverbindlich in welfischen Besitz überführt worden wäre; wozu es allerdings nicht kam, weil der Kaiser dieses Ansinnen ablehnte. Nach Übernahme der hannoverschen Regierung hat Ernst August die von Spinola ausgehenden Reunionsgespräche weiterhin gefördert. Es sei ihm, wie er sich äußerte, dasjenige, was zu beforderung des Unions-Werks dienen mag, nicht misfällig. ${ }^{3}$ Mit diesen Gesprächen federführend beauftragt war auf hannoverscher Seite der Konsistorialrat und Abt von Loccum Gerard Wolter Molanus, ein Schüler des Helmstedter Theologieprofessors Georg Calixts, des wichtigsten Vertreters einer irenischen Theologie im lutherischen Protestantismus des 17. Jahrhunderts. ${ }^{4}$ Auch Gottfried Wilhelm Leibniz beteiligte sich an diesen kirchenpolitischen und theologischen Gesprächen, in denen teilweise äußerst subtile theologische Materien erörtert wurden. ${ }^{5}$ Herzog Ernst August konnte

2 Zu den Reunionsgesprächen siehe Hans Otte / Richard Schenk (Hg.), Die Reunionsgespräche im Niedersachsen des 17. Jahrhunderts. Rojas y Spinola - Molan - Lebniz (Studien zur Kirchengeschichte Niedersachsens 37), Göttingen 1999; zu den konfessionellen Interessen der welfischen Herzöge im 17. Jahrhundert siehe Hans Otte, Glaubenswechsel, Reichpolitik und Wiedervereinigung der Kirchen. Der Beginn der Reunionsverhandlungen in Hannover und die Interessen der welfischen Herzoge, in: Ebd., S. 56-84.

3 Zit. nach Otte, 1999 (wie Anm. 2), S. 79.

4 Heinz Weidemann, Gerard Wolter Molanus, Abt zu Loccum. Eine Biographie, Bd. 2, Göttingen 1929; zu Georg Calixt siehe die Artikel von Inge Mager, in: Religion in Geschichte und Gegenwart. Handwörterbuch für Religion und Geschichtswissenschaft, Bd. 2,Tübingen 42008, Sp. 12f. und von Johannes Wallmann, in: Theologische Realenzyklopädie, Bd. 7, S.552-559; Martin Ohst, Gerard Wolter Molanus und seine Stellung zum Projekt einer kirchlichen Reunion, in: Heinz Duchhardt / Gerhard May (Hg.), Union - Konversion - Toleranz. Dimensionen der Annäherung zwischen den christlichen Konfessionen im 17. und 18. Jahrhundert (Veröffentlichungen des Instituts für Europäische Geschichte Mainz, Beiheft 50), Mainz 2000, S. 171-197.

5 Weidemann, 1929 (wie Anm. 4), S. 76ff, 142; Hartmut Rudolph, Bemerkungen zu Leibniz' Reunionskonzept, in: Duchhardt / May, 2000 (wie Anm. 4), S. 227-242. 
an diesen seit 1688 neu aufgenommenen Gesprächen nur gelegen sein, sekundierten sie doch die Verhandlungen seiner Geheimen Räte in Wien, welche sich dort zur selben Zeit um die Erlangung der Kurwürde für Hannover bemühten. Im Gegensatz zu Molanus und Leibniz war Ernst August an den theologischen Inhalten der Unionsgespräche aber in keiner Weise interessiert, ihm ging es ausschließlich um politische Vorteile für seine Dynastie und sein Territorium. Um seine Aussichten auf die neunte Kur zu erhöhen, hatte Ernst August wohl zeitweilig sogar einen Konfessionswechsel ins Auge gefasst, zumindest wurde dieser in Wien als möglich angenommen. ${ }^{6}$ Politische Zugeständnisse an den Kaiser waren zwar notwendig, wie zum Beispiel die Zusage, bei künftigen Kaiserwahlen stets für den Habsburger Kandidaten zu stimmen, eine Konversion jedoch nicht. ${ }^{7}$ Im Dezember 1692 wurde Ernst August von Kaiser Leopold I. zum Kurfürsten erhoben. ${ }^{8}$

Weniger aus politischen denn aus Vernunft- und Toleranzgründen hat die gebildete Kurfürstin Sophie den Reunionsbestrebungen, wie sie zu ihrer Zeit in Hannover diskutiert wurden, etwas abgewinnen können. ${ }^{9}$

Ich hoffe, alle Christen werden bald eins sein [...]. In jener Welt wird man uns nicht fragen, von welcher Religion wir gewesen sein, sondern was wir Gutes und Böses getan haben; daran ist wohl am meisten gelegen, das andere ist ein Pfaffengezänk, das bei den Fürsten stehet zu akkordieren. ${ }^{10}$

Und an anderer Stelle betont die in Glaubensfragen tolerante Sophie: Die Uneinigkeit der Christen sei eine Schande. Bei ihr diene ein Türke, der wolle erst ein Christ werden, wenn wir in unserem christlichen Glauben eins seien. ${ }^{11}$ Sophie hatte auch keine Bedenken, ihrer Nichte Lieselotte von der Pfalz zwecks Heirat des Herzogs Philipp von Orléans, des Bruders Ludwig XIV., den Übertritt zum Katholizismus zu empfehlen. ${ }^{12}$

Am Hofe in Hannover herrschte eine Kirchlichkeit aus Staatsräson. Ernst August besuchte wohl regelmäßig den Gottesdienst, las aber während der Predigt durchaus

6 Georg Schnath, Geschichte Hannovers im Zeitalter der neunten Kur und der englischen Sukzession 1674-1714, Bd. 1, Hildesheim 1938, S. 488.

7 Zu den Verhandlungen über die hannoversche Kurwürde siehe Schnath, 1938 (wie Anm. 6), S. 592-650, bes. 633-535.

8 Die Zustimmung des Reichstags ließ allerdings noch auf sich warten.

9 Mathilde Knoop, Kurfürstin Sophie von Hannover (Niedersächsische Biographien 1), Hannover 1999, S.139-145.

10 Brief an die Raugräfin Luise (1661-1733), die Tochter ihres Bruders Karl Ludwig aus zweiter, morganatischer Ehe, in: Robert Geerds (Hg.), Die Mutter der Könige von Preußen und England: Sophie von Hannover. Memoiren und Briefe, übersetzt von dems., München / Leipzig 1913, S. 219.

11 Eduard Bodemann (Hg.), Briefe der Kurfürstin Sophie von Hannover an die Raugräfinnen und Raugrafen zu Pfalz, Leipzig 1888, S. 91.

12 Ragnhild Hatton, Georg I. Ein deutscher Kurfürst auf Englands Thron, Frankfurt/Main 21985, S. 74. 
auch Komödien, und manchmal so laut, dass sich seine Frau Sophie gestört fühlte beim Briefeschreiben. ${ }^{13}$ Bei einer solch indifferenten Einstellung zur Religion verwundert es nicht, dass der Kurfürstin Sophie der Gedanke eher fern lag, das englische Erbe aus konfessionellen Gründen anzutreten. Wie ihren Briefen zu entnehmen ist, schien ihr das Erbe ohnehin nicht von jedem Makel ganz frei zu sein. Den Pretender Jakob III., dessen Porträt bei ihr im Schloss in Hannover hing, schien sie zuweilen wohl doch als rechtmäßigen Erben des britischen Königreichs anzusehen. Wenn sie dem englischen Erbe überhaupt etwas abgewinnen konnte, dann war es die Aussicht auf machtpolitischen Zugewinn für ihre Nachkommen und ihr Haus. ${ }^{14}$

Nachdem die hannoversche Kurwürde gesichert war und schließlich auch der Reichstag 1708 der Erhebung Hannovers in den Kurfürstenstand zugestimmt hatte, musste auf Rom und den Kaiser keine besondere Rücksicht mehr genommen werden. Vielmehr galt es nun, da die englische Sukzession in greifbare Nähe gerückt war, alle möglichen Irritationen im Vorfeld zu vermeiden. Solche waren noch einmal aufgetreten, als Maximilian Wilhelm, der Bruder Georg Ludwigs und dritte Sohn Ernst Augusts, gerade in jener Zeit zum Katholizismus konvertierte, als in England über die hannoversche Thronfolge verhandelt wurde. Die hannoverschen Gesandten in Den Haag und London empfahlen deshalb, Hannover müsse sich jetzt stärker protestantisch zeigen, um die Thronfolge der Welfen in England nicht zu gefährden. ${ }^{15}$

Georg Ludwig, an dessen Hof, wie es heißt. „die frivolen Kurtisanen den Ton bestimmten " ${ }^{16}$ präsentierte sich fortan öffentlich als Verteidiger des Protestantismus und antizipierte damit gewissermaßen, was bei einer Thronbesteigung in England von ihm erwartet wurde. Jetzt wurde keine besondere Toleranz gegenüber den Katholiken mehr geübt wie noch bei seinen beiden Vorgängern Johann Friedrich und Ernst August. ${ }^{17}$ Vielmehr erließ er am 25. April 1713 ein Religionsedikt, das den Status der Katholiken im Kurfürstentum rechtlich regelte und die Ausübung ihrer Religion stark einschränkte. ${ }^{18}$

13 Eduard Bodemann, Herzogin Sophie von Hannover, in: Wilhelm Maurenbrecher (Hg.), Historisches Taschenbuch, Sechste Folge, Siebenter Jahrgang, Leipzig 1888, S. 29-86, hier: S. 81.

14 Gerd van den Heuvel, „Kein Königreich ist mit einer guten Gesundheit zu vergleichen.“ Liselotte von der Pfalz kommentiert die hannoversche Sukzession, in: Heide Barmeyer (Hg.), Hannover und die englische Thronfolge (Hannoversche Schriften zur Regional- und Lokalgeschichte 19), Bielefeld 2005, S. 141-156, hier: S. 153.

15 Hatton, 1985 (wie Anm. 12), S. 74.

16 Joachim Lampe, Aristokratie, Hofadel und Staatspatriziat in Kurhannover: die Lebenskreise der höheren Beamten an den kurhannoverschen Zentral- und Hofbehörden 1714-1760, Göttingen 1963, S. 134.

17 Hans-Georg Aschoff, Die Reunionsgespräche zwischen Katholiken und Protestanten im 17. Jahrhundert, in: Barmeyer, 2005 (wie Anm. 14), S. 179-197, hier: S. 179f.

18 Aschoff, 2005 (wie Anm. 17), S. 180. 
II.

Als Kurfürst Georg Ludwig den englischen Thron bestieg, war er für die Engländer ein weitgehend unbeschriebenes Blatt. Aus ihrer Sicht handelte es sich um einen Fürsten aus einem eher unbedeutenden deutschen Territorium, mit dem sie eigentlich nur eines verband: der Protestantismus. But now, God be prais'd, though our present most gracious Sovereign is a Foreigner, yet is he a Protestant.$^{19}$ So heißt es in einer anonymen Schrift aus dem Jahr seines Regierungsantritts. Der Protestantismus war, wie Frauke Geyken formuliert, „das Brennglas, durch das das Bild Georgs Formen annahm“. ${ }^{20}$ Auf jeden Fall gehörte es fortan zur Selbststilisierung des englischen Königs, sich als Retter und Wahrer protestantischer Interessen darzustellen, und so wurde seinen Titeln der des Defender of Faith hinzugefügt. ${ }^{21}$

Da Georg I. den Gegnern der hannoverschen Sukzession anfangs kaum Angriffsflächen bot, geriet nun seine Konfession in den Fokus der Kritik. ${ }^{22}$ Gewiss, der neue König war ein Protestant, aber er war ein Lutheraner. Konnte aber ein Lutheraner das Oberhaupt der Kirche von England werden?

Kurz nach der Thronbesteigung entstand eine öffentliche Debatte über diese Frage, die auf zwei Ebenen geführt wurde. ${ }^{23}$ Zum einen wurde gefragt, ob die theologischen Grundpositionen der anglikanischen und der lutherischen Kirche überhaupt übereinstimmten; zum anderen wurden die äußere Form des Gottesdienstes und die Ausgestaltung des Kirchenraums verglichen, um dadurch Unterschiede oder Ähnlichkeiten zwischen den beiden Konfessionen festzustellen. Während die Befürworter der hannoverschen Sukzession betonten, dass die Lutheraner wahre Protestanten seien, es eine Gemeinsamkeit in fundamentalen Glaubensartikeln gäbe und die Verwandtschaft zwischen der lutherischen und der anglikanischen Liturgie überaus deutlich sei, hoben die Kritiker gerade signifikante Unterschiede hervor; und einige dieser Kritiker sahen gar eine gewisse Nähe der lutherischen Kirche zum Katholizismus. Letzteres war eine gefährliche Sichtweise, weil damit den Jakobiten ein Argument in die Hände gespielt wurde, welches den Ausschluss der katholischen Stuarts von der Erbfolge wieder in Frage zu stellen vermochte. Deshalb sah sich der lutherische Pre-

19 Zit. nach Frauke Geyken, Gentlemen auf Reisen: das britische Deutschlandbild im 18. Jahrhundert, Frankfurt/Main 2002, S. 135.

20 Geyken, 2002 (wie Anm. 19), S. 136.

21 Geyken, 2002 (wie Anm. 19), S. 200; Andrew C. Thompson, The confessional dimension, in: Brendan Simms (Hg.), The Hanoverian dimension in British History, Cambridge 2007, S. 161-182, hier: S. 170.

22 Geyken, 2002 (wie Anm. 19), S. 208.

23 Hannah Smith, Georgian Monarchy. Politics and Culture, 1714-1760, Cambridge 2006, S. 48 weist auf diese Debatte hin. Etwas ausführlicher geht Andrew Spicer, Introduction: Lutheran Churches and Confessional Identy, in: Ders. (Hg.), Lutheran Churches in Early Modern Europe, Ashgate 2012, S. 1-4, auf diese Debatte ein. 
diger an der Londoner Trinitatiskirche und spätere Hofprediger in Hannover, Balthasar Mentzer, veranlasst, eine Schrift zur Verteidigung der lutherischen Lehre gegen den Vorwurf der Papisterei herauszugeben. ${ }^{24}$ Diese kurz nach der Thronbesteigung Georg I. entstandene Debatte zeitigte jedoch keine nachhaltige Wirkung; denn die Kritiker verstummten recht bald. Georg I. hielt sich - wie es für ihn verpflichtend war - aus Gründen der Staatsräson an die anglikanische Kirche.

\section{III.}

Die hannoversche Sukzession in England hat eine weitere, zu Beginn des 18. Jahrhunderts nur noch glimmende religions- und kirchenpolitische Debatte neu zu entfachen vermocht. Im Jahr 1714 erschien in London in zweiter Auflage die Übersetzung des "Jus feciale divinum“ von Samuel Pufendorf. ${ }^{25}$ Pufendorf wirkte seit 1688 als Hofhistoriograph und Geheimer Rat am brandenburg-preußischen Hof in Berlin. Dass dieses von Theophilus Dorrington ${ }^{26}$ ins Englische übersetzte Buch Pufendorfs, dessen erste Auflage bereits 1703 in England erschienen war, ${ }^{27}$ gerade zu diesem Zeitpunkt erneut herausgegeben wurde, war kein Zufall. Der veränderte Titel der zweiten Auflage gibt nicht nur das Anliegen des Buches wieder, nämlich die Prinzipien des Luthertums im Hinblick auf deren Vereinbarkeit mit der englischen Kirche zu untersuchen, sondern nennt auch den Anlass: die Thronbesteigung Georgs I. Der Titel der Schrift lautete: „A View of the principles of the Lutheran churches, shewing how far they agree with the Church of England: being a seasonable essay towards the uniting of Protestants upon the accession of His Majesty King George to the throne of these kingdoms. “28 Die Tatsache, dass ein Lutheraner nun zum Oberhaupt der anglikani-

24 Balthasar Mentzer, A vindication of the Lutheran Religion, from the charge of Popery, London 1720 . Zu Mentzer siehe Religion in Geschichte und Gegenwart. Handwörterbuch für Religion und Geschichtswissenschaft, Bd. 5, Tübingen 42002, Sp. 1104; Rudolf Steinmetz, Die Generalsuperintendenten von Calenberg, in: Zeitschrift der Gesellschaft für Niedersächsische Kirchengeschichte 13 (1908), S. 25-267, hier: S. $153-166$.

25 Zur Entstehung und zum Kontext des Jus feciale divinum siehe die Einleitung von Detlef Döring in der von ihm herausgegebenen historisch-kritischen Edition dieser Schrift im Rahmen der Gesammelten Werke Samuel Pufendorfs: Bd. 9: Jus feciale divinum, Berlin 2004.

26 Zu Dorringtons Kontakten nach Deutschland und zu dessen Übersetzung der Pufendorf-Schrift siehe Alexander Schunka, Zwischen Kontingenz und Providenz. Frühe Englandkontakte der Halleschen Pietisten und protestantische Irenik um 1700, in: Pietismus und Neuzeit. Ein Jahrbuch zur Geschichte des neueren Protestantismus 34 (2008), S. 82-114, hier: S. 91-94, $106 f$.

27 Samuel Pufendorf, The divine feudal law: or, covenants with mankind, Represented. Together with means for The uniting of Protestants. In which also the principles of the Lutheran Churches are stated and defended, By Samuel Baron Pufendorf. Translated from the Latin by Theophilus Dorrington, London 1703. Schon diese erste Übersetzung war im Kontext der erwarteten hannoverschen Sukzession erschienen.

28 London 1714. 
schen Kirche geworden war, und er damit in seiner Person gewissermaßen eine innerprotestantische Union verkörperte, beflügelte die Anhänger des Unionsgedankens zu verstärktem Engagement, nachdem in den Jahrzehnten zuvor erste entsprechende Initiativen im Sande verlaufen waren.

In Europa hatte sich um 1700 im Protestantismus ein Gefühl der Bedrohung verbreitet, veranlasst durch die zunehmende Bedrängung der Protestanten in den habsburgischen Ländern, durch die Aufhebung des Edikts von Nantes 1685, die zu einer Massenauswanderung der Hugenotten aus Frankreich führte, durch die Konversion des sächsischen Kurfürsten zum Katholizismus 1697 und schließlich durch den von Ludwig XIV. ausgelösten neunjährigen Krieg, der eine Rekatholisierung der von den Franzosen besetzten Gebiete zur Folge hatte. Vor dem Hintergrund dieser Entwicklungen gab es Kräfte in allen protestantischen Konfessionen, die für einen engeren politischen und vor allem kirchenpolitischen Zusammenschluss der Protestanten plädierten. Der Bischof von Lincoln, William Wake ${ }^{29}$, betonte in einer Predigt anlässlich des ersten Jahrestages der Thronbesteigung am 1. August 1715 vor König Georg I. die Notwendigkeit einer Union zwischen den protestantischen Kirchen und Staaten, wobei die anglikanische Kirche seiner Ansicht nach als the head of the Protestant interest die Führungsrolle zufalle. ${ }^{30}$ Als Wake ein Jahr später zum Erzbischof von Canterbury berufen wurde, ergriff er selbst die Initiative und suchte Mitstreiter für seine Unionspläne. ${ }^{31}$ In Hannover fand er diese im Abt von Loccum, Gerard Wolter Molanus, und in Gottfried Wilhelm Leibniz. Beiden war die Einheit der Christenheit - und wie bereits erwähnt, durchaus auch unter Einschluss des Katholizismus - seit Jahren ein Anliegen; es ging ihnen nun vor allem um eine Wiedervereinigung der infolge der Reformation entstandenen Kirchen. Mit dem reformierten Hofprediger Daniel Ernst Jablonski in Berlin hatten Molanus und Leibniz schon intensiv, allerdings vergeblich über eine innerprotestantische Union verhandelt. ${ }^{32}$ Große Hoffnungen ruhten nun auf dem Erzbischof von Canterbury, der über den einflussreichen hannoverschen Minister Andreas Gottlieb von Bernstorff, ${ }^{33}$ dem ersten Leiter der

29 Norman Sykes, William Wake. Archbishop of Canterbury 1657-1737, 2 Bde, Cambridge 1957.

30 Sykes, 1957 (wie Anm. 29), Bd. 2, S. 1.

31 Sykes, 1957 (wie Anm. 29), Bd. 2, S. 3-22; Wolf-Friedrich Schäufele, Erzbischof William Wake von Canterbury (1657-1737) und die Einigung der europäischen Christenheit, in: Duchhardt / May, 2000 (wie Anm. 4), S. 301-314.

32 Weidemann, 1929 (wie Anm. 4), Bd. 2, S. 130-147.

33 Hermann Kellenbenz, Andreas Gottlieb von Bernstorff, in: Neue Deutsche Biographie, Bd. 2, Berlin 1955, S.137f.; Hartwig Graf von Bernstorff, Andreas Gottlieb von Bernstorff 1649-1726. Staatsmann, Junker, Patriarch. Zwischen deutschem Partikularismus und europäischer Politik, Bochum 1999. 
Deutschen Kanzlei in London, Einfluss auf Georg I. zu nehmen versuchte. ${ }^{34}$ Trotz wiederholter Bemühungen gelang es Wake jedoch nicht, konkrete Ergebnisse zu erzielen und mit Bernstorffs Rückkehr nach Hannover verlor er schließlich seinen wichtigsten Mittelsmann zum König. Dass die Unionsbefürworter ihre Hoffnung auf Wake gesetzt hatten, geschah wohl in Verkennung seiner wahren kirchenpolitischen Position. Denn für Wake war die anglikanische Kirche die vollkommenste protestantische Kirche und an diese sollten sich die anderen Kirchen anpassen. Eine Einigung zwischen der anglikanischen Kirche und den protestantischen Kirchen auf dem Kontinent war auch deshalb sehr schwierig, weil die Kirche von England auf ihre Episkopalverfassung und die apostolische Sukzession nicht verzichten wollte. ${ }^{35}$ Gewissermaßen als kleine Lösung einer solchen Union wurde die Vereinigung der Kirchen innerhalb der Personalunion kurzzeitig ins Auge gefasst. Dem Vorbild des hannoverschen Königs folgend könnten, so der Vorschlag, doch zumindest die anglikanische Kirche Englands und die lutherische Kirche Hannovers sich vereinen und damit eine einheitliche Staatskirche im Raum der Personalunion geschaffen werden. ${ }^{36}$ Das war allerdings eine Idee, die am Londoner Hof niemals ernsthaft in Erwägung gezogen wurde.

Parallel zu diesen über einzelne Personen laufenden Bemühungen um eine innerprotestantische Union nahmen sich seit 1717, dem Jahr des Reformationsjubiläums, ebenfalls Gesandte des Corpus Evangelicorum auf dem immerwährenden Reichstag in Regensburg der Sache der Protestanten an. ${ }^{37}$ Mit dem hannoverschen Reichstagsgesandten Johann Rudolf von Wrisberg, der die Interessen Hannovers und Georgs I. auf dem Reichstag in Regensburg vertrat, standen sowohl der Erzbischof von Canterbury als auch der Abt von Loccum in Briefwechsel..$^{38}$ Nach langen Verhandlungen, bei denen die Gesandten der beiden aufstrebenden protestantischen Mächte Brandenburg-Preußen und Hannover den Takt vorgaben, einigten sich die Gesandten der protestantischen Länder im Februar 1722 auf ein „Conclusum wegen näherer Zusammensetzung oder Vereinigung der Protestierenden“. Diese Vereinbarung sah die gegenseitige Anerkennung von Lutheranern und Reformierten als Glaubensbrüder vor. Sie fand allerdings keineswegs den Beifall aller lutherischen Kirchen. Den

34 Sykes, 1957, (wie Anm. 29), Bd. 2, S. 69-72; William O’Reilly, Protestantische Kultur in England und Irland im 17. und 18. Jahrhundert, in: Peter Claus Hartmann (Hg.), Religion und Kultur im Europa des 17. und 18. Jahrhunderts (Mainzer Studien zur Neueren Geschichte 12), Frankfurt/Main u.a. 2004, S. 57-72, hier: bes. S. 57-61.

35 O’Reilly, 2004 (wie Anm. 34), S. 10f.; Schäufele, 2000 (wie Anm. 31), S. 213.

36 Sykes, 1957 (wie Anm. 29), Bd. 2, S. $61 \mathrm{f}$.

37 Wolf-Friedrich Schäufele, Christoph Matthäus Pfaff und die Kirchenunionsbestrebungen des Corpus Evangelicorum 1717-1726 (Veröffentlichungen des Instituts für Europäische Geschichte Mainz 172), Mainz 1998, S. 87-123.

38 Schäufele, 1998 (wie Anm. 37), S. 195. 
heftigsten Widerstand leistete die Hamburger Geistlichkeit, allen voran der dortige Hauptpastor Erdmann Neumeister, dessen Pamphlete in ganz Deutschland Aufsehen erregten. ${ }^{39}$ Einige Verse aus dem „Neumeisterlichen Madrigal auf die Union“ (1722) seien im Folgenden zitiert: ${ }^{40}$

Was vor ein Wunder-Ding will allererst entstehn?

Des Teuffels Mutter soll anjetzo schwanger gehn,

Man sagt daßsie ihr Wochen-Bette

$\mathrm{Zu}$ Regensburg längst aufgeschlagen hätte

Und wäre schon im Kreissen.

Gebiert sie einen jungen Sohn,

So soll er Syncretismus heissen,

Wirds aber eine Tochter seyn,

So heists sie Union;

Jedoch geräth das Werck nicht etwa noch ins Stecken,

So schwör ich Stein und Bein:

Es wird die Mutter sammt der Brut verrecken.

Das Werk geriet, wie Neumeister hoffte, tatsächlich ins Stecken. In den folgenden Jahren zeigte sich, dass weder der König noch die hannoverschen Minister an dem Einigungswerk wirklich interessiert waren. ${ }^{41}$ Infolgedessen verzichtete man in Hannover auf dessen Ratifizierung, worauf diese dann auch in Preußen und anderen protestantischen Territorien unterblieb. ${ }^{42}$ Die innerprotestantischen Unionsbestrebungen waren damit auch auf dem Reichstag zu Regensburg endgültig gescheitert.

Die Verabschiedung des Act of Settlement, die darauf folgende hannoversche Sukzession in England und die sich daran anschließenden religions- und kirchenpolitischen Debatten und Gespräche zeigen, welche Bedeutung selbst drei Generationen nach dem Westfälischen Frieden die Religion als Faktor der Politik noch hatte, wenn auch zunehmend nur noch als rhetorisches Mittel zur Durchsetzung politischer Interessen und zur Legitimierung politischen Handelns. ${ }^{43}$ Religion bleibt als Faktor innenwie außenpolitischer Maßnahmen auch noch im 18. Jahrhundert, wie Andrew C.

39 Schäufele, 1998 (wie Anm. 37), S. 302. Zu Erdmann Neumeister siehe die Artikel von Johannes Wallmann in: Religion in Geschichte und Gegenwart, Bd. 6, Tübingen 42003, Sp. 231 und Wolfgang Miersemann in: Neue Deutsche Biographie, Bd. 19, Berlin 1999, S. 170f.

40 Zit. nach Schäufele, 1998 (wie Anm. 37), S. 321.

41 Schäufele, 1998 (wie Anm. 37), S. $284 f$.

42 Schäufele, 1998 (wie Anm. 37), S. 287.

43 Vgl. Manfred Schlenke, England blickt nach Europa. Das konfessionelle Argument in der englischen Politik in der Mitte des 18. Jahrhunderts, in: Paul Kluke/ Peter Alter (Hg.), Aspekte der deutsch-britischen Beziehungen im Laufe der Jahrhunderte (Veröffentlichungen des Deutschen Historischen Instituts London 4), Stuttgart 1978, S. 24-45. 
Thompson zu Recht betont, eine „soft power“ im politischen Geschäft. ${ }^{44}$ Schließlich bleibt zu betonen, dass die Reunionsbemühungen zwischen Katholiken und Protestanten wie auch die innerprotestantischen Gespräche über eine Union nur ein erstes Wetterleuchten einer neu anbrechenden Zeit größerer Toleranz darstellten. Der Weg von einer tolerantia ecclesiastica zu einer wirklichen Union bzw. Reunion der durch die Reformation getrennten Kirchen ist - sehen wir einmal von der preußischen Union des 19. Jahrhunderts $\mathrm{ab}$ - allerdings bis heute eine noch weitgehend unbewältigte Aufgabe geblieben.

\section{IV.}

Der religiösen Indifferenz der beiden ersten hannoverschen Könige auf dem englischen Thron ist es wohl vor allem zuzuschreiben, dass sich am Hof in London, im Zentrum der aufstrebenden Weltmacht, eine religiöse Eigenkultur etablieren konnte, ${ }^{45}$ wie es am Hof in Hannover zu jener Zeit nicht möglich gewesen wäre. Georg von Dänemark, der Ehemann der späteren Königin Anne, hatte 1700 in London eine lutherische Hofkapelle gegründet, die mitten im St James Palace eingerichtet wurde. Die Aufsicht über diese Kapelle lag in der Hand des Bischofs von London, welcher auch die vom König ernannten Hofprediger einsetzte. Bei jeder Thronbesteigung mussten diese vom König jeweils neu bestätigt werden. ${ }^{46}$ Auf Empfehlung des Sekretärs des Prinzen Georg, Heinrich Wilhelm Ludolf, wurde als erster Hofprediger der aus der Grafschaft Waldeck gebürtige Anton Wilhelm Böhme berufen. Böhme war ein Pietist hallescher Provenienz, ein Schüler August Hermann Franckes. ${ }^{47}$ Mit seiner Berufung hatte die pietistische Bewegung, welche die größte Frömmigkeitsund Erneuerungsbewegung innerhalb des Protestantismus seit der Reformation war, einen ersten festen Stützpunkt in der angelsächsischen Welt erlangt. Dieser Schritt war von Bedeutung, weil der Hallesche Pietismus weitgespannte Zielsetzungen verfolgte. August Hermann Francke ging es, wie er in einer programmatischen Schrift schrieb, um eine reale Verbesserung in allen Ständen in und ausserhalb Teutschlands, ja in Europa und allen übrigen Theilen der Welt. ${ }^{48}$ Weltverwandlung durch Menschen-

44 Thompson, 2007 (wie Anm. 21), S. 170.

45 Manfred Jakubowski-Tiessen, Eigenkultur und Traditionsbildung, in: Hartmut Lehmann (Hg.), Geschichte des Pietismus, Bd. 4: Glaubenswelt und Lebenwelten, Göttingen 2004, S. 195-210.

46 Daniel L. Brunner, Halle pietists in England. Anthony William Boehm and the Society for Promoting Christian Knowledge (Arbeiten zur Geschichte des Pietismus 29), Göttingen 1993, S. 49.

47 Zu Böhme siehe Brunner, 1993 (wie Anm. 46); Arno Sames, Anton Wilhelm Böhme (1673-1722). Studien zum ökumenischen Denken und Handeln eines hallischen Pietisten (Arbeiten zur Geschichte des Pietismus 26), Göttingen 1990.

48 So der nicht ganz vollständige Titel des sogenannten „Universalprojekts“, in: Erhard Peschke (Hg.), 
verwandlung war das Ziel des Pietismus. ${ }^{49}$ Welcher Ort hätte im 18. Jahrhundert als Ausgangspunkt derartiger globaler Zielsetzungen besser geeignet sein können als die Weltstadt London.

Bemerkenswert ist, dass Georg I. bei seinem Regierungsantritt den deutschen Hofprediger Böhme trotz seiner pietistischen Gesinnung in seinem Amt bestätigte. In Halle wurde dies als ein ermutigendes Zeichen gedeutet. Nur kurze Zeit später bekam Böhme von dort einen Vorschlag, auf welche Weise er den englischen König möglicherweise dazu bewegen könne, dem Hallischen Pietismus künftig besondere Förderung zu erweisen. Böhme solle auf das Beispiel des preußischen Königs und dessen Protektion für die Halleschen Unternehmungen hinweisen. Wenn Böhme bei seinem Vorgehen erfolgreich wäre, würde es, wie betont wurde, nicht allein ein Segen für England sein, sondern auch der pietistischen Sache in Hannover helfen. Georg I. erwies sich für ein solches Ansinnen wie für pietistische Anliegen überhaupt als wenig zugänglich; derartige Bemühungen scheiterten vor allem an seinem religiösen Desinteresse. ${ }^{50}$

Als Böhme 1722 verstarb und damit die Stelle des deutschen Hofpredigers vakant wurde, war den halleschen Pietisten klar, dass für dessen Nachfolge nur ein Theologe aus dem Kurfürstentum Hannover in Frage käme. Wie konnte man dennoch die pietistische Tradition in der Londoner Hofkapelle sichern? Dies könne nur gelingen, wie August Hermann Francke sehr wohl wusste, wenn man einen Pietisten aus dem Kurfürstentum finden würde, der für dieses Amt die notwendige Qualifikation mitbrächte. In einer geheim gehaltenen Aktion gelang es Francke über das pietistische Netzwerk in die entscheidenden Gremien in London und Hannover den Namen eines pietistischen Kandidaten zu lancieren, der dann auch als Nachfolger Böhmes berufen wurde: ${ }^{51}$ Es war Friedrich Michael Ziegenhagen, seit 1718 Kabinettsprediger des Grafen Ernst August von Platen (1674-1726) in Linden bei Hannover. ${ }^{52}$ Im November 1722 trat Ziegenhagen sein Amt als Hofprediger in London an, ein Amt, das er über 50 Jahre bis zu seinem Lebensende 1776 innehatte. Wie sein Vater so hatte auch Georg II. bei seinem Regierungsantritt den deutschen Hofprediger in seinem Amt belassen. Auf diese Weise konnte der hallesche Pietismus in den folgenden Jahrzehnten im Schatten der englischen Weltmacht seine Beziehungsnetze von London

August Hermann Francke, Werke in Auswahl, Berlin 1969, S. 108-115.

49 Manfred Jakubowski-Tiessen, Von Spener zu Francke - Der Pietismus im 17. und 18. Jahrhundert in seiner europäischen Dimension, in: Holger Zaunstäck / Thomas Müller-Bahlcke / Claus Veltmann (Hg.), Die Welt verändern. August Hermann Francke. Ein Lebenswerk um 1700, Halle 2013, S. 29-39.

50 Brunner, 1993 (wie Anm. 46), S. 56.

51 Brunner, 1993 (wie Anm. 46), S. 57.

52 Zur Biographie Friedrich Michael Ziegenhagens siehe Christina Jetter-Staib, Halle, England und das Reich Gottes weltweit - Friedrich Michael Ziegenhagen (1694-1776). Hallescher Pietist und Londoner Hofprediger (Hallesche Forschungen 34), Halle 2013. 
aus bis in die außereuropäische Welt spannen. Die lutherische Hofkapelle mit dem Hofprediger Ziegenhagen und seinen ebenfalls pietistischen Mitarbeitern wurde zur wichtigsten Schaltstelle für alle von Halle ausgehenden Missionsaktivitäten in Nordamerika und Indien. ${ }^{53}$ Hier wurden die auszusendenden Missionare und Prediger, wie etwa auch Heinrich Melchior Mühlenberg, ${ }^{54}$ vor ihrer Abreise betreut und auf den Dienst in den fremden Kontinenten vorbereitet. ${ }^{55}$ Über Ziegenhagen lief nahezu alle Post zwischen Halle, England, Indien und Nordamerika. Die lutherische Hofkapelle war zugleich der Ort, an dem sich eine Schar religiös Gleichgesinnter aus dem Kreis der in London ansässigen Deutschen versammelte, um eine pietistische Gemeinschaft zu gegenseitiger Erbauung und religiöser Stärkung zu pflegen. ${ }^{56}$ Ziegenhagens Ziel war es, diesen Kreis frommer deutscher Landsleute durch seine regelmäßige Predigttätigkeit und Seelsorge zusammenzuhalten und möglichst zu erweitern. ${ }^{57}$

In den fünf Jahrzehnten seines Londoner Wirkens nahm Ziegenhagen eine Schlüsselstelle ein für die Verbindung zu einflussreichen Kreisen in England, die bereit waren die missionarischen Aktivitäten Halles finanziell zu unterstützen. Besonders wichtig war seine Verbindung zu der 1698 gegründeten Society for Promoting Christian Knowledge, einer Institution, die ähnliche Anliegen wie die Pietisten vertrat, nämlich die Menschen durch christliche Unterweisung zu einer religiösen und moralischen Erneuerung zu führen. ${ }^{58}$ In dieser Gesellschaft, der die beiden Hofprediger Böhme und Ziegenhagen eng verbunden waren, fanden die pietistischen Unterneh-

53 Norman J. Threinen, Friedrich Ziegenhagen: the London Connection to India and America, in: Hans-Jürgen Grabbe (Hg.), Halle Pietism, Colonial North America, and the Young United States (USA-Studien 15), Stuttgart 2008, S. 113-134. Über die Mitarbeiter Ziegenhagens und das Umfeld der Hofkapelle siehe Jetter-Staib, 2013 (wie Anm. 52), S. 87-90, S. 136-144. Nachfolger Ziegenhagens wurde sein Mitarbeiter, der Lektor Friedrich Wilhelm Pasche, ebenfalls Hallischer Pietist, der u. a die transatlantische Korrespondenz fortsetzte.

54 Jetter-Staib, 2013 (wie Anm. 52), S. 348-370; zu Mühlenberg siehe den jüngst erschienen Sammelband Hermann Wellenreuther / Thomas Müller-Bahlcke / A. Gregg Roeber (Hg.), The Transatlantic World of Heinrich Melchior Mühlenberg in the Eighteenth Century (Hallesche Forschungen 35), Halle 2013.

55 Jetter-Staib, 2013 (wie Anm. 52), S. 217-225.

56 Die gründliche Erforschung der deutschen Hofkapelle und ihrer Gemeinde bleibt ein wichtiges Desiderat der Forschung.

57 Die vor ihrem Ehemann geflüchtete Gräfin Johanna Sophie von Schaumburg-Lippe, die mit der kurfürstlichen Familie 1714 nach London übersiedelte und dort bis 1728 bei der Prinzessin Wilhelmine Karoline, der Frau des Kurprinzen, als Oberhofmeisterin diente, hat der pietistischen Gemeinschaft der deutschen Hofkapelle in London angehört und dort ein Bekehrungserlebnis gehabt. Nach dem Tod ihres Ehemannes, des Grafen Friedrich Christian, und der Übernahme der Regierung durch ihren Sohn Albrecht Wolfgang, kehrte Johanna Sophie nach Schaumburg-Lippe zurück und hat dort mit großem Engagement die pietistische Bewegung gefördert. Siehe Manfred Jakubowski-Tiessen, Religiosität in Krisenzeiten. Der Pietismus in Schaumburg-Lippe, in: Helge Bei der Wieden (Hg.), Die Ausstrahlung der Reformation. Beiträge zu Kirche und Alltag in Nordwestdeutschland (Studien zur Kirchengeschichte Niedersachsens 43), Göttingen 2011, S. 73-86.

58 Zur Society for Promoting Christian Knowledge siehe Brunner, 1993 (wie Anm. 46), S. 101-197. Sowohl Böhme als auch Ziegenhagen waren in führenden Positionen in dieser Missionsgesellschaft. 
mungen auf den außereuropäischen Missionsfeldern immer wieder hilfreiche Förderung. Ziegenhagen wurde zu einer wichtigen Instanz innerhalb des internationalen pietistischen Netzwerkes und wusste seine herausgehobene Stellung, die noch durch seinen direkten Zugang zur Königsfamilie eine zusätzliche Aufwertung erfuhr, für die Sache des Pietismus zu nutzen. ${ }^{59}$ In England und am Hof wurde er durchaus als ein Vertreter des Halleschen Pietismus wahrgenommen. Obwohl er selbst weder in Indien noch in Amerika gewesen war, empfand er seine Londoner Tätigkeit stets als einen Beitrag zur Ausbreitung des Reichs Gottes auf Erden.

\section{V.}

Während den Pietisten am Hofe in London weitgehende Freiheiten für ihre religiösen Ambitionen eingeräumt wurden, wurde der Pietismus im Kurfürstentum Hannover von Regierung und kirchlicher Oberbehörde als deviante religiöse Bewegung beurteilt, die es zu bekämpfen galt. Bereits unter dem Kurfürsten Georg Ludwig war 1703 ein erstes Edikt gegen den Pietismus erlassen worden, das sich gegen Leute richtete, welche mit sogenannten pietistischen auch anderen neuerlichen Irrthümern in Religions-Sachen behafftet sind. ${ }^{60}$ Das Edikt verbot jegliche Separation vom öffentlichen Gottesdienst und vom Abendmahl. Vier Wochen später wurde zur Erläuterung des Edikts an alle Geistlichen ein von Molanus entworfenes Konsistorialschreiben versandt, worin die Merkmale der Pietisterey im Einzelnen beschrieben werden. ${ }^{61}$ Nachrichten über eine neu entstandene radikalpietistische Bewegung im Harz, die inzwischen an das Konsistorium in Hannover gelangt waren, ${ }^{62}$ haben den Inhalt dieses Konsistorialschreibens wohl mit bestimmt. Molanus wollte in seinem Konsistorialschreiben vermutlich vor allem die Merkmale des separatistischen Pietismus beschreiben; seine „Notae charactericae pietisticae“ wurden vom hannoverschen Konsistorium aber zur allgemeinen Charakterisierung des Pietismus, eben auch des kirchlichen herangezogen. Molanus fehlte, wie sein Biograph Heinz Weidemann betont, eine

59 Jetter-Staib, 2013 (wie Anm. 52), S. 436.

60 Rudolf Ruprecht, Der Pietismus des 18. Jahrhunderts in den Hannoverschen Stammländern, Göttingen 1919, S. 18f.; Manfred Jakubowski-Tiessen, Der Pietismus in Niedersachsen, in: Martin Brecht / Klaus Deppermann (Hg.), Geschichte des Pietismus, Bd. 2: Der Pietismus des 18. Jahrhunderts, Göttingen 1995, S. 428f.

61 Ruprecht (wie Anm. 60), S. 19f.; Jakubowski-Tiessen, 1995 (wie Anm. 60), S. $428 f$.

62 Ruprecht, 1919 (wie Anm. 60), S. 16ff., 28f.; Manfred Jakubowski-Tiessen, Religiöse Konflikte und soziale Proteste. Bergarbeiterunruhen und radikalpietistische Bewegungen im Oberharz im 18. Jahrhundert, in: Jahrbuch der Gesellschaft für Niedersächsische Kirchengeschichte 94 (1996), S. 123-138. 
seelsorgerische Gabe; deshalb habe er „die Tiefe dieser Bewegungen nicht zu begreifen vermocht, sondern allein vom grünen Tische aus sein Urteil über sie gefällt" ${ }^{63}$

Die radikalpietistischen separatistischen Bewegungen im Harz, die zu ersten Auseinandersetzungen mit den kirchlichen Instanzen führten, haben das Bild des Pietismus in den welfischen Ländern in den folgenden Jahrzehnten hauptsächlich geprägt und immer wieder staatliche Reaktionen hervorgerufen. Die zum Teil harten staatlichen Maßnahmen gegen diese religiösen Bewegungen erklären sich teilweise daraus, dass die nach primär absolutistischen Grundsätzen herrschende Regierung des Kurfürstentums durch die separatistischen Bewegungen das einheitliche landesherrliche Kirchenwesen gefährdet sah. Diese Sorge kommt in dem Pietistenedikt vom 15. Mai 1711 zum Ausdruck, in dem der regelmäßige Besuch des Gottesdienstes unter Androhung der Landesverweisung zur Pflicht erklärt und die Konventikel bei Gefängnisstrafe erneut verboten wurden. Die Berufung auf den inneren Christus könne, wie es in dem Edikt ferner heißt, letztlich alle Ordnungen der Kirche aufheben, Tür und Tor für mancherlei Irrtümer öffnen und dazu führen, daß ein jeder nach seinem Gutdüncken und Wahn sich eine Religion fingiren möge. ${ }^{64}$ Die Regierung hat das Verhalten der Harzer Pietisten schließlich „als Ungehorsam gegen staatliche Ordnungen gebrandmarkt und demgemäß als Aufruhr gegen den Staat bestraft “ ${ }^{65}$

Dass sich ein kirchlicher Pietismus trotz des schroff antipietistischen Kurses des Konsistoriums und der harten antipietistischen Edikte dennoch im Kurfürstentum hat entwickeln können, lag vor allem daran, dass einige einflussreiche Adlige des Kurfürstentums sich der pietistischen Bewegung angeschlossen hatten. Seit 1727 fanden die Hallischen Pietisten in dem Staatsminister Gerlach Adolph von Münchhausen (1688-1770), den Begründer der Göttinger Universität, einen Protektor in der Hannoverschen Regierung. ${ }^{66}$ Als König Georg II. 1727 auf den Thron gelangte, berief er Münchhausen in das Geheime Ratskollegium und ernannte ihn 1732 zugleich zum Großvogt der cellischen Amtsvogteien. Münchhausen plädierte für Toleranz in religiösen Fragen, lehnte fundamentalistische Positionen ab und versuchte stets eine Vermittlerrolle in Religionsstreitigkeiten einzunehmen. ${ }^{67}$ Er war zwar selbst kein Pietist,

63 Weidemann, 1929 (wie Anm. 4), Bd. 1, S. 98.

64 Ruprecht, 1919 (wie Anm. 60), S. 39.

65 Ruprecht, 1919 (wie Anm. 60), S.40.

66 Walter Buff, Gerlach Adolph Freiherr von Münchhausen als Gründer der Universität Göttingen, Göttingen 1937.

67 An die Theologische Fakultät der neu gegründeten Universität in Göttingen wollte A. G. v. Münchhausen nur solche Theologen berufen, die sich in den Auseinandersetzungen zwischen der lutherischen Orthodoxie und dem Pietismus nicht besonders exponiert hatten. Die Fakultät sollte eine irenische theologische Ausrichtung bekommen. Emil F. Rössler, Die Gründung der Universität Göttingen. Eine Sammlung bisher ungedruckter Entwürfe, Berichte und Briefe, Göttingen 1855, S. 422ff. 
zeigte aber eine gewisse Aufgeschlossenheit gegenüber den Pietisten, ${ }^{68}$ was wohl nicht zuletzt daran lag, dass seine Frau Wilhelmine Sophie, geb. von Wangenheim (17031750), eine bekennende Pietistin war. Sie hielt in ihrem Haus regelmäßig pietistische Konventikel und pflegte weit gespannte Beziehungen zu führenden Pietisten ihrer Zeit. ${ }^{69}$ Besonders intensiv waren ihre Kontakte nach Halle und Wernigerode. Zwar nahm ihr Ehemann an den Konventikeln in seinem Haus nicht teil, war jedoch für die erweckten Besucher seiner Frau stets zu sprechen und unterhielt sich auch bei den gemeinsamen Mahlzeiten gern mit ihnen über erbauliche Themen. Sein Wohlwollen für die Pietisten brachte ihn in eine gewisse Opposition zum orthodoxen Konsistorium. ${ }^{70}$

Dennoch gelang es dem pietistenfeindlichen Konsistorium im Jahr 1740, König Georg II. davon zu überzeugen, den ihrer Ansicht nach immer noch virulenten Harzer Separatismus mit einem weiteren scharfen Edikt zu bekämpfen. Das noch im selben Jahr erlassene Pietisten-Edikt stellte den letzten Versuch zur Bekämpfung der separatistischen Bewegung im Harz dar. ${ }^{71}$ Noch einmal wurden lokale Untersuchungen und Maßnahmen gegen einzelne Pietisten eingeleitet. Zu jener Zeit hielten viele Pfarrer der Harzregion das Edikt jedoch für unnötig und eher kontraproduktiv, da die radikalpietistische Bewegung bereits am Abklingen war und es ihnen nun vor allem darum ging, die Separatisten unter das Dach der Kirche zurückzuholen.

Während der pietistischen Bewegung unter den hannoverschen Königen Georg I. und Georg II. in London relativ große Entfaltungsmöglichkeiten eingeräumt wurden, sahen sich die Pietisten in Kurhannover starken staatlichen und kirchlichen Repressalien ausgesetzt. Wie lässt sich dieses asymmetrische obrigkeitliche Handeln gegenüber dem Pietismus im Raum der Personalunion erklären? Zunächst ist zu bedenken, dass die beiden ersten hannoverschen Könige religiösen Fragen und theologischen Erörterungen kein allzu großes Gewicht beigelegt haben. Religion war zwar noch ein Faktor der Politik und diente durchaus noch zur Legitimierung politischen Handelns, aber die eigenen Glaubenswelten der Könige blieben davon zumeist unberührt. Ihre religiöse Indifferenz und ihr Desinteresse an theologischen Materien zeitigte ein eher geringes Engagement in kirchlichen Angelegenheiten. Der dadurch entstandene Spielraum wurde in London und Hannover auf unterschiedliche Weise genutzt. Die deutschen Hofprediger Böhme und Ziegenhagen und ihre Mitarbeiter in der deutschen Hofkapelle nutzten diesen, um die pietistische Bewegung mit ihrem globalen Missionsanliegen zu fördern. In Hannover dagegen hat das Konsistorium sich diesen Spielraum zunutze gemacht und sich aller ihm zur Verfügung stehenden

68 Buff, 1937 (wie Anm. 66), S. 15.

69 Ruprecht, 1919 (wie Anm. 60), S. 135 f.

70 Ruprecht, 1919 (wie Anm. 60), S. 137.

71 Ruprecht, 1919 (wie Anm. 60), S. $96 f f$. 
Möglichkeiten bedient, um die Pietisten und den Pietismus in Kurhannover zu bekämpfen, weil es in der pietistischen Bewegung eine große Gefahr für die evangelische Kirche sah.

Als weitere Ursache für die Divergenz im obrigkeitlichen Verhalten gegenüber dem Pietismus in London und Hannover sind die unterschiedlichen religionspolitischen Verhältnisse in diesen Ländern zu berücksichtigen. Im Unterschied zu England, wo sich auf der Grundlage der Bill of Rights ein parlamentarisches Regierungssystem herausgebildet hatte, herrschten in Kurhannover noch absolutistische Strukturen vor. Dabei bildete die konfessionelle Uniformität eine zusätzliche ideologische Klammer für das absolutistische Herrschaftssystem. In England hatte sich dagegen eine konfessionelle Pluralität entwickelt, seitdem der 1689 unter Wilhelm von Oranien vom Parlament verabschiedete Toleration Act den englischen Freikirchen die freie Religionsausübung gestattete. Auch ausländischen Religionsgemeinschaften war die freie Religionsausübung in London erlaubt. In England war dadurch eine religiöse Vielfalt entstanden, die gegenseitige Toleranz erforderte und einen höheren Grad an Freiheit gewährte, was auch der deutschen Hofkapelle und ihren Predigern zu Gute kam, die keiner hannoverschen Oberbehörde, sondern dem Bischof von London unterstanden.

Und schließlich war die von Ziegenhagen unterstützte missionarische Tätigkeit der Pietisten auf den fernen Missionsfeldern in Indien und Nordamerika für das expandierende Britische Weltreich eine wohl eher willkommene als unerwünschte Aktivität, flankierte sie doch die politische, wirtschaftliche und kulturelle Expansion in den außereuropäischen Besitzungen, selbst wenn die Königsfamilie „nur zu begrenzten Unterstützungsleistungen für die Mission bereit" war und Bitten um Spendenbeiträge sogar ablehnte. ${ }^{72}$

\section{Epilog}

Trotz seiner jahrzehntelangen erfolgreichen Mitarbeit am Aufbau des Reichs Gottes auf Erden musste auch Ziegenhagen erfahren, dass es auch für den Pietismus Grenzen des Wachstums gab. Nicht jede Person, die sich ihm anvertraute, konnte er für die Sache des Pietismus gewinnen. Um Ostern 1741 kam beispielsweise der später berühmte Göttinger Professor der Orientalistik, Johann David Michaelis, aus Halle mit einer Empfehlung zu Ziegenhagen nach London. Ziegenhagen scheint einige Hoffnungen auf diesen jungen begabten Theologen gesetzt zu haben, der während seines etwa siebzehnmonatigen Aufenthalts in London auf Bitten Ziegenhagens vierzehntägig die Nachmittags- und manchmal auch die Morgenpredigten in der Hofkapelle

72 Jetter-Staib, 2013 (wie Anm. 52), S. 248. 
übernahm. Nach Michaelis eigener Aussage habe er bei seinem dortigen Aufenthalt von Ziegenhagens theologischen Kenntnissen sehr profitieren können, obgleich dieser, wie Michaelis in seiner Lebensbeschreibung hervorhebt, ein Pietist und noch dazu ein ziemlich eifriger war. ${ }^{73}$ Schon in der Zeit seines Englandaufenthalts registrierten Ziegenhagen und sein Kollege Samuel Theodor Albinus bei Michaelis mit einiger Sorge eine gewisse Beeinflussung durch die Bibelwissenschaft des englischen Deismus. Etwa fünfzehn Jahre später äußerte Albinus, dass er einen sehr betrübten Begriff von der Universität Göttingen und inspecie von dem vormals so redlichen M. Mich[aelis] gewonnen hätte. Er könne kaum glauben, so stellte Albinus in einem Brief mit großer Verwunderung fest, daß Michaelis ein Religions-Spötter solte worden seyn. ${ }^{74}$ Aber das war natürlich eine Beurteilung aus pietistischer Perspektive.

73 Johann David Michaelis, Lebensbeschreibung von ihm selbst abgefaßt, mit Anmerkungen von Hassencamp, Rinteln / Leipzig 1793, S. 36.

74 Albinus an Sebastian Andreas Fabricius, dem Organisator der Missionsabteilung in Halle, 8.9.1756, Archiv der Franckeschen Stiftungen: I D 4:42.2; zit. bei Jetter-Staib, 2013 (wie Anm. 52), S. 182. 